\title{
High-power broadly tunable picosecond IR laser system for use in nonlinear spectroscopic applications
}

\author{
D. E. Gragson, B. M. Mc Carty, and G. L. Richmond \\ Department of Chemistry, University of Oregon, Eugene, Oregon 97403 \\ D. S. Alavi \\ Shared Laser Facility, University of Oregon, Eugene, Oregon 97403
}

Received December 5, 1995; revised manuscript received March 21, 1996

\begin{abstract}
We developed a high-power tunable picosecond IR laser system suitable for nonlinear spectroscopic investigations. We employ a Ti:sapphire regenerative amplifier that produces $1.9-\mathrm{ps}, 17-\mathrm{cm}^{-1}, 800-\mathrm{nm}$ pulses at $1 \mathrm{kHz}$ as a pump source. White-light generation in ethylene glycol and optical parametric amplification in potassium titanyl phosphate are used to produce the IR pulses. The tuning range extends from 2.4 to $3.8 \mu \mathrm{m}$ in the idler and 1.0 to $1.2 \mu \mathrm{m}$ in the signal. A total efficiency (signal plus idler) as high as $20 \%$ was achieved. The spatial, spectral, and temporal characteristics of the IR beam are presented along with vibrational sumfrequency spectra from a glass/octadecyltrichlorosilane/air interface. (C) 1996 Optical Society of America.
\end{abstract}

\section{INTRODUCTION}

The study of interfaces buried between two condensed phases has grown rapidly in the past decade. Nonlinearoptical methods such as second-harmonic generation and sum-frequency generation (SFG) are techniques that have made significant contributions in this area. For the case of IR-visible SFG, one can achieve both interfacial and molecular selectivity and thus study an interface on a molecular level. ${ }^{1-6}$ Crucial to the development of these nonlinear optical methods has been the development of nanosecond tunable IR laser systems necessary to perform SFG experiments. The development of picosecond higher-peak-power tunable IR laser systems has been somewhat slower. One reason is that the IR pulses must have both high peak power and a narrow spectral bandwidth so that different vibrational modes can be resolved. Systems producing nanosecond pulses have narrow bandwidths but low peak power, whereas systems producing femtosecond pulses have high peak power but large bandwidths. We developed a high-peak-power tunable IR laser system that is optimized with respect to both of these limitations. It is based on a Ti:sapphire regenerative amplifier that produces $1.9-\mathrm{ps}, 17-\mathrm{cm}^{-1}, 800-\mathrm{nm}$ pulses at a repetition rate of $1 \mathrm{kHz}$. The conversion of the $800-\mathrm{nm}$ pulses into tunable high-power IR pulses is achieved through optical parametric amplification of a white-light continuum.

Parametric processes such as optical parametric generation and optical parametric amplification (OPA) in nonlinear crystals have been used extensively to generate tunable IR pulses. ${ }^{7,8}$ In each of these processes a photon pair is created at the expense of a pump photon with the wavelengths governed by the phase-matching conditions in the nonlinear crystal and the wavelength of the pump photon. By convention, the shorter-wavelength component is termed the signal and the longer-wavelength component termed the idler. The majority of these systems are based on Nd:YAG pump lasers and produce pulses with durations from tens of picoseconds to nanoseconds and bandwidths from several wave numbers to a few tenths of a wave number. ${ }^{9-12}$ More recently, regeneratively amplified Ti:sapphire lasers have been used to produce subpicosecond tunable IR pulses with bandwidths of the order of 100 wave numbers. ${ }^{13-15}$ In fact there are some commercially available systems that produce tunable femtosecond IR pulses. These systems, however, are not particularly well suited to nonlinear spectroscopic applications owing to the large bandwidths and the low pulse energies produced. Further, the majority of these systems use $\beta$-barium borate or lithium triborate for the nonlinear crystal, which limits the tuning range to 2.4 $\mu \mathrm{m}$. We employ a Ti:sapphire regenerative amplifier that produces 1.9-ps pulses at $800 \mathrm{~nm}$ and a bandwidth of $17 \mathrm{~cm}^{-1}$ as our pump source. The IR generation is based on white-light-continuum generation in ethylene glycol and OPA in two independently pumped potassium titanyl phosphate (KTP) crystals. This technique has been developed in the femtosecond regime $\mathrm{e}^{11,16-18}$ but has not been exploited in the smaller-bandwidth picosecond regime. White-light generation was chosen to provide an intense seed covering the entire signal wavelength region, 1-1.6 $\mu \mathrm{m}$. Our first attempt at IR generation involved seeding the first KTP crystal with the entire IR portion of the continuum from $1 \mu \mathrm{m}$ to $1.6 \mu \mathrm{m}$. This, however, produced signal and idler bandwidths in excess of $100 \mathrm{~cm}^{-1}$, with the large bandwidth being a result of the large acceptance bandwidth of KTP in the 2.6-3.8 $\mu \mathrm{m}$ region. The bandwidth can be narrowed by supplying the KTP crystal with a seed that is narrower than the acceptance 


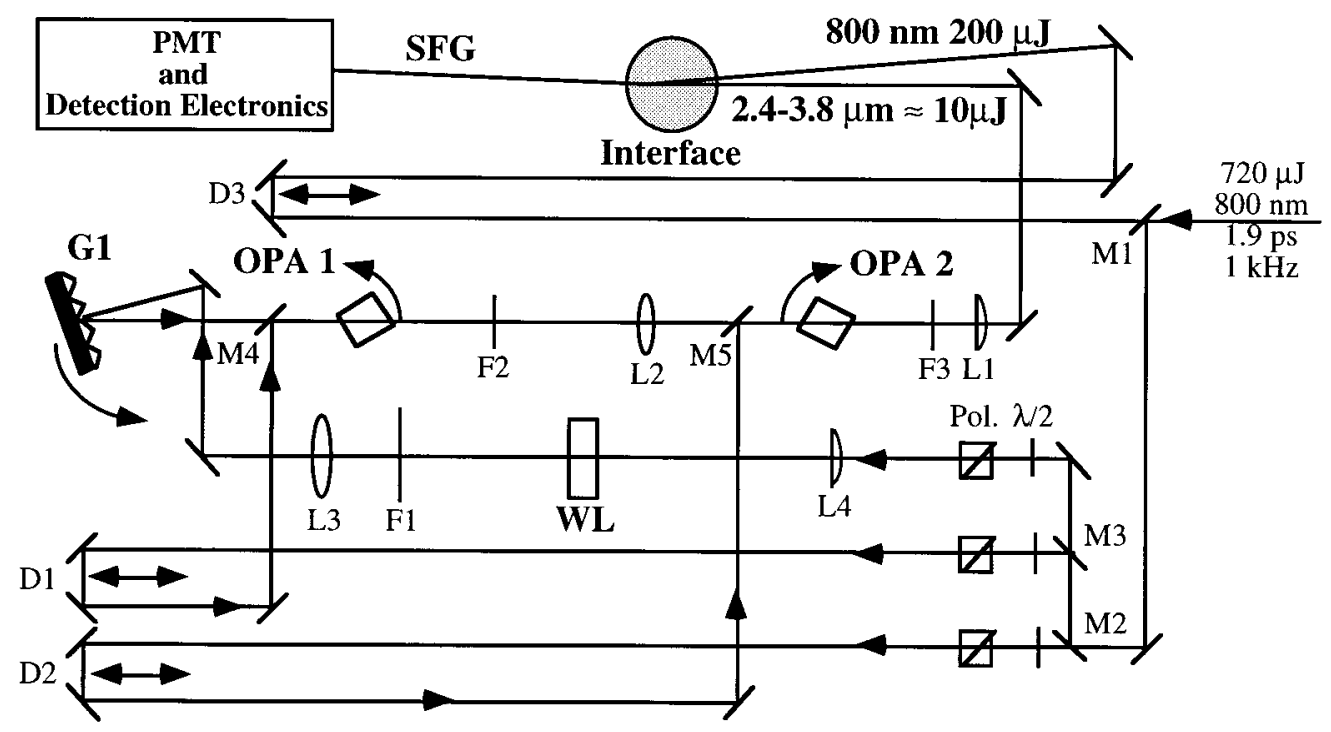

Fig. 1. Optical layout of a tunable IR laser system (see text for description).

bandwidth of the crystal. We accomplished this by using a grating to disperse the continuum spatially before seeding the first KTP crystal. With the use of the grating the portion of the IR continuum that actually seeds the first crystal spans approximately $7 \mathrm{~cm}^{-1}$. Narrowing the output pulses by narrowing the seed pulses before amplification has also been used in the femtosecond regime. ${ }^{11,19}$ KTP was selected as the nonlinear crystal because of the desired tuning range, $1-4 \mu \mathrm{m}$, its figure of merit, which is proportional to the conversion efficiency, and its availability. Further, type II (ordinary idler, extraordinary signal, and ordinary pump) phase matching in the $x z$ plane of the KTP crystals was chosen because of a higher $d_{\text {eff }}$ and larger tuning range for an 800-nm pump. We use two amplification stages mainly because higher energies can be achieved but also because a second crystal angle tuned in the opposite direction has the effect of compensating for the beam displacement from the first crystal.

\section{EXPERIMENTAL LAYOUT}

The Ti:sapphire regenerative amplifier laser system consists of a Ti:sapphire oscillator (Coherent Mira-900 basic) pumped by an $\mathrm{Ar}^{+}$ion laser (Coherent Innova-310), a stretcher-compressor (Quantronix 4822), and a Ti:sapphire regenerative amplifier (Quantronix 4810) pumped by the second harmonic of a Nd:YLF laser (Quantronix $527 \mathrm{DP}-\mathrm{H})$. The output of the laser system (720 $\mu \mathrm{J}, 800$ $\mathrm{nm}$ ) is first split with a partially reflecting mirror (M1) that sends $460 \mu \mathrm{J}$ to the optics for nonlinear conversion and leaves $260 \mu \mathrm{J}$ for the SFG experiments. Figure $1 \mathrm{de}-$ picts the optical setup used to convert the 800 -nm photons into IR photons. This $460 \mu \mathrm{J}$ is split into three pump lines with two 50\% reflecting mirrors, M2 and M3. The three lines are used for pumping the white-light generator WL and the two KTP $(5 \mathrm{~mm} \times 5 \mathrm{~mm} \times 5 \mathrm{~mm})$ crystals OPA1 and OPA2. For each line a polarizer/halfwave-plate combination is used to select the polarization and power in that line. The white-light pump passes through lens L4, which focuses $110 \mu \mathrm{J}$ of the 800-nm light into the ethylene glycol. The ethylene glycol flows through a $10 \mathrm{~mm} \times 10 \mathrm{~mm} \times 50 \mathrm{~mm}$ quartz cell (WL) that limits the effect of bubble formation on the white light generated by boiling of the ethylene glycol. The bubble formation is further minimized by cooling the ethylene glycol to $16{ }^{\circ} \mathrm{C}$. The white light generated then passes through filter F1 (RG-850), which transmits only the IR portion of the continuum and blocks the visible and the remaining 800-nm pump. The IR portion of the continuum is brought to focus at the position of OPA1 by lens L3. The bandwidth of the seed-supplied OPA1 is narrowed by grating $\mathrm{G} 1(600 \mathrm{~g} / \mathrm{mm}$, blazed at $750 \mathrm{~nm})$. The white-light seed, approximately $7-\mathrm{cm}^{-1}$ bandwidth, is then amplified in OPA1 with $120 \mu \mathrm{J}$ of the $800-\mathrm{nm}$ light telescoped to a beam diameter, $2 \omega$, of $355 \mu \mathrm{m}(64$ $\mathrm{GW} / \mathrm{cm}^{2}$ ). The IR seed and the 800 -nm pump are combined with dichroic mirror M4, which reflects $800 \mathrm{~nm}$ and transmits 1-1.6- $\mu \mathrm{m}$ light. The temporal overlap of the seed and the pump in OPA1 is controlled by delay line D1, which incorporates a hollow retroreflector. After this first stage of amplification, signal energies as high as 10 $\mu \mathrm{J}$ and idler energies as high as $2.5 \mu \mathrm{J}$ are obtained. Since higher energies are desired, another amplification stage (OPA2) located $560 \mathrm{~mm}$ from OPA1 is employed. Filter F2 (80\% transmission from 2.2-4.1 $\mu \mathrm{m})$ is used to block the amplified signal and the $800-\mathrm{nm}$ pump and to transmit the idler for amplification in OPA2. The idler is used for the seed in OPA2 because higher energies and better spatial beam profiles are attained in this configuration (see the following discussion). The idler from OPA1 is focused to a beam diameter, $2 \omega$, of $760 \mu \mathrm{m}$ at the position of OPA2 by a $\mathrm{CaF}_{2}$ lens, L2. The idler generated in OPA1 is amplified in OPA2 with $160 \mu \mathrm{J}$ of the $800-\mathrm{nm}$ light, which is telescoped to a beam diameter, $2 \omega$, of 598 $\mu \mathrm{m}\left(30 \mathrm{GW} / \mathrm{cm}^{2}\right)$. The idler and the $800-\mathrm{nm}$ pump are combined with the use of dichroic mirror M5 that reflects 
$800 \mathrm{~nm}$ and transmits $1.5-4.0 \mu \mathrm{m}$. The temporal overlap of the seed and the pump in OPA2 is controlled by a second delay line D2. One of two filters is used in the position of F3: the first for the measurement of the idler energy (80\% transmission from 2.2-4.1 $\mu \mathrm{m}$ ) and the other for the measurement of the signal energy (RG1000). The IR output from OPA2 is then collimated with lens L1 for the SFG experiments. Except for the $\mathrm{CaF}_{2}$ lenses, L1 and L2, all other lenses are broadband antireflection coated BK7, and all mirrors not mentioned are either broadband near-IR high reflectors for the $800 \mathrm{~nm}$ or protected gold for the IR.

The pump laser system and the IR laser system were characterized in terms of power, wavelength, spectral bandwidth, temporal pulse width, and spatial beam quality. Wavelengths were measured with a $0.3-\mathrm{m}$ crossed Czerny-Turner monochromator with a 300 grooves $/ \mathrm{mm}$ grating blazed for $3 \mu \mathrm{m}$ and an indium antimonide (InSb) detector. Bandwidths [full width at half-maximum intensity (FWHM)] were measured with the monochromator or with a grating (1200 or 1800 grooves $/ \mathrm{mm}$ ) in combination with a one-dimensional CCD array (UniData BP2048). The monochromator was used in different orders of diffraction depending on the wavelength of light being examined. Specifically, the pump-beam, the signal-beam, and the idler-beam spectra were obtained in fourth-, third-, and first-order diffraction, respectively. Further, the resolution of the monochromator was approximately $3 \mathrm{~cm}^{-1}$ at the pump and the signal wavelengths and approximately $1 \mathrm{~cm}^{-1}$ at the idler wavelengths. The resolution of the grating/CCD combination was approximately $4 \mathrm{~cm}^{-1}$ and was mainly limited by the beam diameter. Powers were measured with a pyroelectric joulemeter (Molectron J3-09), and temporal pulse profiles were characterized by autocorrelations (Inrad 5-14B) and/or cross correlations (FWHM).

Spatial beam quality was characterized by knife-edge measurements across the transverse profile of the beams. Assuming an approximately Gaussian transverse spatial profile, the distance between knife-edge positions passing $16 \%$ and $84 \%$ of the total beam energy was used to approximate the Gaussian beam radius $\omega$, which has the standard definition of the half-width at $1 / e$ of maximum for the field amplitude, or the half-width at $1 / e^{2}$ of maximum for the intensity. ${ }^{20}$ This is in turn related to the FWHM for the intensity by $\mathrm{FWHM}=(2 \ln 2)^{1 / 2} \omega$ $=1.177 \omega$. The measured pump- and idler-beam sizes were then used to estimate the $M^{2}$ parameter $^{21}$ (i.e., times diffraction limited) for the pump and the idler beams.

\section{Ti:SAPPHIRE LASER CHARACTERIZATION}

A titanium:sapphire (Ti:sapphire) regenerative amplifier system based on the chirped pulse amplification technique is used as the pump source and thus is a primary determinant of the spectral, the spatial, and the temporal characteristics of the output of the tunable IR system. It is therefore quite important that those same spectral, spatial, and temporal characteristics of the regenerative amplifier pump source be well understood.
The grating-based stretcher employed in the chirped pulse amplification technique is a particularly convenient location for manipulation of the spectral, and therefore temporal, properties of the amplifier seed pulses. At the midpoint of the stretcher the various wavelength components are spatially dispersed, allowing the possibility of spectral filtering. Other researchers have used sophisticated amplitude and phase masks specifically to tailor the amplified pulses in an attempt to control coherently the optically driven processes. ${ }^{22,23}$ For the present application, all that is required is a simple reduction of the bandwidth. This is accomplished by the insertion of an aperture at the midpoint of the stretcher, limiting the seed bandwidth passed to the amplifier by the stretcher. This technique must be used with caution, however. The stretching process relies on the temporal chirping of a wide spectral bandwidth. If this bandwidth is narrowed, the corresponding temporal width of the chirped pulse also becomes narrower, leading to higher peak powers in the amplifier. (In contrast to the transform-limited case, in which pulse width and bandwidth are inversely related, pulse width and bandwidth are linearly related in the highly chirped case for a given amount of dispersion.) The grating pulse stretcher must be modified to provide greater dispersion in order to stretch these spectrally narrower pulses to a safely amplified pulse width. This can be most easily accomplished by insertion of a different grating with a higher groove density and therefore greater pulse stretching per unit bandwidth.

The use of an aperture at the midpoint of the pulse stretcher, although simple to implement, requires care in analyzing pulse-width and bandwidth data of the amplified pulses. For our purposes, output pulses with bandwidths of $20 \mathrm{~cm}^{-1}$ or less are desired from seed pulses that are $\sim 100-\mathrm{cm}^{-1}$ FWHM. Over the central $20 \mathrm{~cm}^{-1}$ of the seed bandwidth, the spectral intensity distribution is relatively flat, leading to an essentially rectangular spectrum for the amplifier output. Such a spectrum yields pulses with an intensity profile $I(t) \propto(\sin t)^{2} / t^{2} \equiv \operatorname{sinc}^{2} t$. The time-bandwidth product for this temporal profile is 0.886 (Ref. 24), which is significantly different from the values of more commonly used functional forms, such as Gaussian (0.441) or $\operatorname{sech}^{2}(0.315)$. In addition, pulse widths are measured by autocorrelation techniques that yield a temporal function that has widths that are proportional to the underlying intensity profile. The constant of proportionality varies depending on the functional form of the temporal intensity profile. The ratio of autocorrelation width to pulse width (both FWHM) is 1.33 for sinc ${ }^{2}$ pulses, in contrast to 1.41 for Gaussian pulses and 1.55 for $\operatorname{sech}^{2}$ pulses. For the conversion of autocorrelation width to pulse width in instances where there is no basis for choice of a functional form, an error of less than $10 \%$ is introduced by use of 1.44 for this ratio (the midpoint of the $1.33-1.55$ range).

Temporal and spectral measurements were made for the Ti:sapphire oscillator output, the stretched seed pulses, and the amplified pulses. The oscillator yielded autocorrelations of 140-fs FWHM, which yields a pulse width of 90-fs FWHM assuming sech ${ }^{2}$ pulses. The corresponding transform-limited spectral bandwidth is 117- $\mathrm{cm}^{-1}$ FWHM. The stretched seed pulses were mea- 

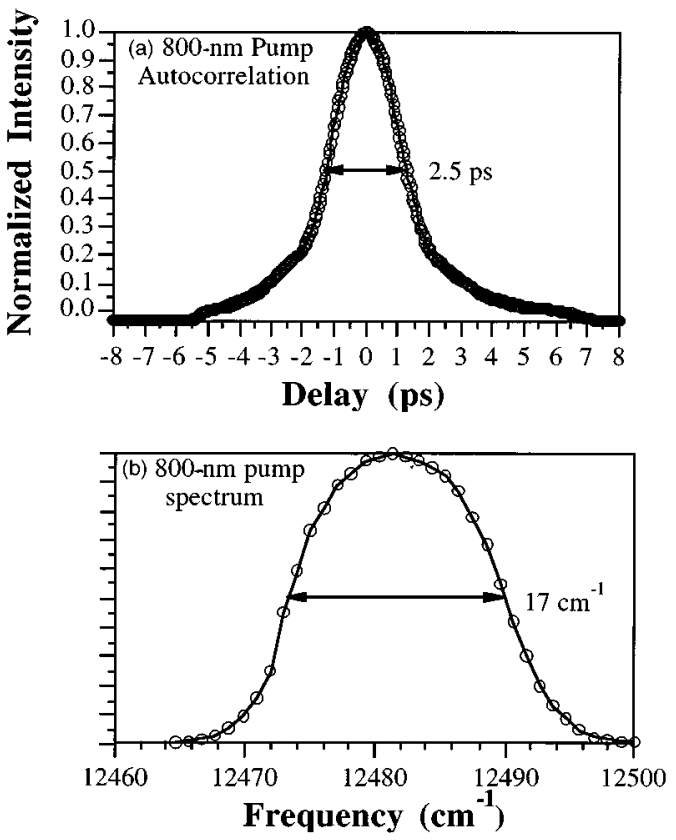

Fig. 2. Autocorrelation and spectrum of the pump (800-nm) beam: (a) autocorrelation and (b) the spectrum obtained with a monochromator (see text). The solid curves are a guide for the eye.

sured with a fast photodiode and a sampling head (Antel AR-S2, Tektronix 7104/S4, 35-ps rise time). In a previous study ${ }^{25}$ describing this system the seed pulse width was measured to be 220-ps FWHM with a bandwidth of $21 \mathrm{~cm}^{-1}$. It appeared essentially rectangular, as one would expect for a rectangular spectrum with a linear chirp. To reduce the bandwidth further in the present version of the apparatus, we used a smaller aperture to restrict the bandwidth further and shorten the stretched pulse to $170 \mathrm{ps}$. This resulted in a bandwidth of $17 \mathrm{~cm}^{-1}$. After amplification, autocorrelation and bandwidth measurements were made of the recompressed pulses, and are shown in Fig. 2. The autocorrelation FWHM was 2.5 $\mathrm{ps}$, resulting in a pulse width of $1.9 \mathrm{ps}$. The bandwidth of $17 \mathrm{~cm}^{-1}$ yields a time-bandwidth product of 0.97 , or 1.1 times transform limited assuming sinc ${ }^{2}$ pulses.

Other methods are under consideration for further narrowing the regenerative amplifier seed spectrum. The rectangular spectrum produced by the hard aperture in the stretcher yields a relatively large time-bandwidth product. One can imagine obtaining higher peak powers for a given bandwidth or smaller bandwidths for a given peak power if a narrowed spectrum could be produced with a smaller time-bandwidth product. Two possible means of achieving this result are (1) the use of a spatially varying filter in place of the hard aperture or (2) the use of a very-narrow-bandpass filter (1-nm FWHM) placed before the stretcher. Either of these could produce a spectrum that was more Gaussian in nature. It should be noted that neither of these eliminates the need for the high-density grating in the stretcher. To push bandwidths even shorter, one must use an even higherdensity grating to obtain suitable pulse stretching before amplification. All of these modifications are under investigation.
The spatial quality of the beam was measured by knifeedge measurements. The compressed output of the regenerative amplifier was thus determined to be 1.2 times diffraction limited.

\section{IR SYSTEM CHARACTERIZATION}

Figure 3 shows the experimental and theoretical tuning curves for type II phase matching in KTP. Theoretical tuning curves were generated by imposing type II (o-ray pump, e-ray signal, and o-ray idler) collinear phase matching in the $x z$ plane $\left(\phi=0^{\circ}\right)$ of the KTP crystal. $^{26}$ The Sellmeier coefficients for KTP used are from Vanherzeele. ${ }^{27}$ The extent of the experimental tuning range is governed by several limitations. First is the transparency region of the KTP crystals, which is $350 \mathrm{~nm}$ to $4.5 \mu \mathrm{m}$. This is the ultimate limitation in that light cannot be generated at wavelengths outside the transparency region. Next is the transmission region of the filters F2 and F3, which is 2.2 to $4.0 \mu \mathrm{m}$ for each. With these filters, idler wavelengths above $4.0 \mu \mathrm{m}$ or below $2.2 \mu \mathrm{m}$ are not transmitted and thus are not available for experiments. The last limitation is the dichroic mirror M4, which reflects $800 \mathrm{~nm}$ and transmits 1.0 to $1.6 \mu \mathrm{m}$. This prevents us from generating light above $4.0 \mu \mathrm{m}$ since wavelengths below $1.0 \mu \mathrm{m}$ are necessary as the signal seed and are not transmitted. From this, one can see that, with the use of different filters and a different dichroic mirror, idler wavelengths to $4.5 \mu \mathrm{m}$ can be achieved.

SFG experiments require high peak intensities for both the visible/near-IR pulse and the tunable IR pulse. In addition, good spatial and temporal overlap must be achieved. These requirements demand good spectral/ temporal pulse characteristics as well as good spatial beam characteristics. The spectral, the temporal, and the spatial characteristics of pump, signal, and idler were all measured and analyzed with these considerations in mind.

The spectral and the temporal characteristics of the signal and the idler pulses produced by the optical para-

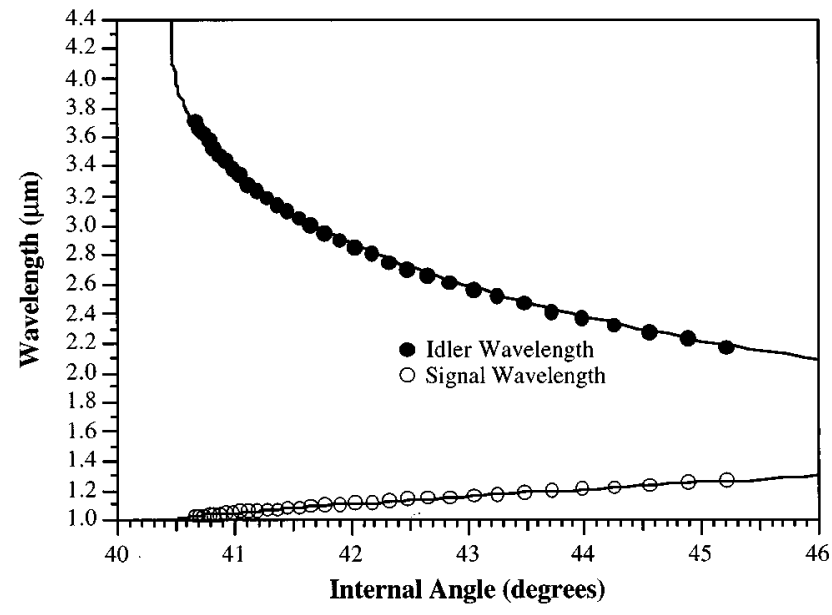

Fig. 3. Tuning curve for KTP OPA under type II phase matching in the $x z$ plane. The markers are the experimental data points for the signal and the idler, the solid curve is the theoretical tuning curve from Eq. (1). 

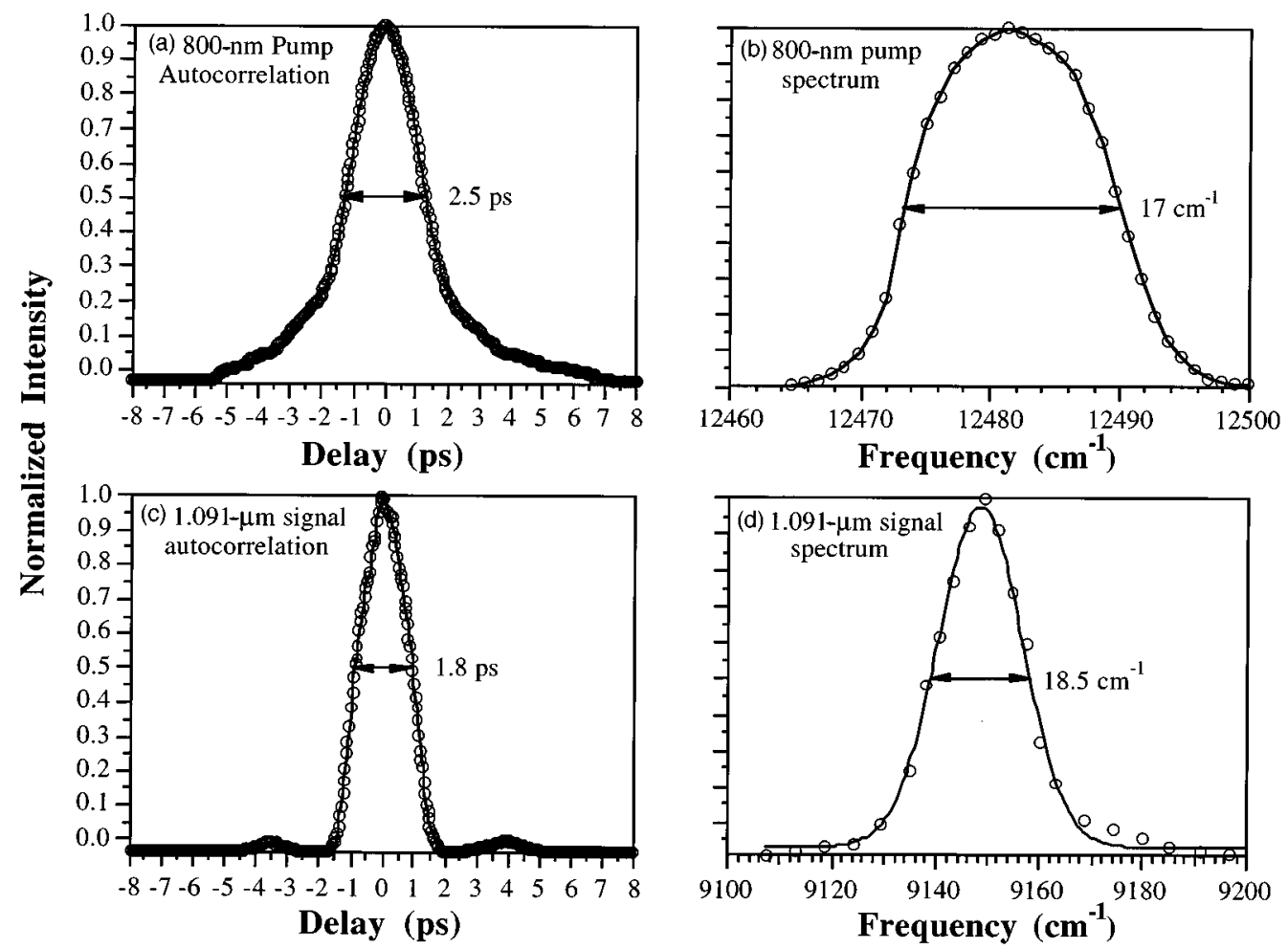

Fig. 4. Autocorrelation and spectra, obtained with a monochromator, of pump (800-nm) and signal (1.091- $\mu \mathrm{m})$ beams: (a) pump autocorrelation, (b) pump spectrum, (c) signal autocorrelation, and (d) signal spectrum. Solid curves are a guide to the eye.

metric amplifiers were measured and compared with those of the pump laser system. Autocorrelations and spectra of both the pump at $800 \mathrm{~nm}$ and the signal at 1.09 $\mu \mathrm{m}$ are shown in Fig. 4. The autocorrelation of the pump beam has a FWHM of $2.5 \mathrm{ps}$, as stated previously, and exhibits the typical shape of an autocorrelation of a sinc ${ }^{2}$ pulse, including slight sidelobes. The corresponding spectrum is flatter on the top than typical spectra of mode-locked lasers, and it is a result of the roughly rectangular spectrum that should result from the hardaperture spectral filter. The spectrum and the autocorrelation of the signal pulses look somewhat different. The signal spectrum is slightly wider than the pump spectrum, which is to be expected, and it looks much more Gaussian in nature than the pump spectrum. A rough calculation of the bandwidth expected to fall within the spatial profile of the pump beam in the first KTP crystal (209- $\mu$ m FWHM) yields

$$
\begin{aligned}
\Delta \bar{\nu} & \approx a \bar{\nu}^{2} \cos \beta\left(\frac{d}{D}\right) \\
& =\frac{\left(9166 \mathrm{~cm}^{-1}\right)^{2}}{\left(6000 \mathrm{~cm}^{-1}\right)} \cos 21^{\circ} \frac{0.209 \mathrm{~mm}}{400 \mathrm{~mm}}=6.8 \mathrm{~cm}^{-1},
\end{aligned}
$$

where $a$ is the groove spacing of the grating, $d$ is the width of the pump beam (FWHM), $D$ is the distance from the grating to the first KTP crystal, and $\beta$ is the angle of diffraction from the grating. The $17-\mathrm{cm}^{-1}$ pump bandwidth yields a lower limit for the signal, and the mea- sured signal bandwidth $\left(18.5 \mathrm{~cm}^{-1}\right)$ is slightly higher than this lower limit. The FWHM of the autocorrelation is significantly narrower than the pump autocorrelation ( 1.8 versus $2.5 \mathrm{ps}$ ), and the sidelobes are much reduced relative to the pump. This would suggest that the functional forms of the signal pulse and the spectrum are quite different from their pump counterparts. An autocorrelation of $1.8 \mathrm{ps}$ implies a pulse width of approximately $1.3 \mathrm{ps}$ (dividing by 1.44) and a time-bandwidth product of 0.72 . This is less than the transform limit for sinc $^{2}$ pulses and approximately twice the transform limit for Gaussian or sech $^{2}$ pulses.

A similar analysis was performed for the idler. However, because of the wavelengths involved $(2.5-3.8 \mu \mathrm{m})$, autocorrelations could not be done with the optics and the nonlinear crystals on hand. Instead, the idler pulses were cross correlated with the pump laser pulses in a KTP crystal by SFG, and a pulse width for the idler was extracted by treating the cross correlation as a convolution of two Gaussians $\left(\Delta t_{c c}^{2}=\Delta t_{p}^{2}+\Delta t_{i}^{2}\right)$. Idler spectra for three different idler wavelengths are shown in Fig. 5, and cross correlation measurements for the same three idler wavelengths are shown in Fig. 6 . At 3.0 and $3.6 \mu \mathrm{m}$ the spectra look quite Gaussian like, and they are nearly the same bandwidth as the pump pulse at 18 and 16 $\mathrm{cm}^{-1}$, respectively. The cross correlations are also well approximated by Gaussians at these two wavelengths with FWHM of $2.3 \mathrm{ps}$ at both wavelengths. Deconvolution of the pump pulse width of $1.9 \mathrm{ps}$ yields idler pulse widths of $1.3 \mathrm{ps}$ and time-bandwidth products of 0.70 and 


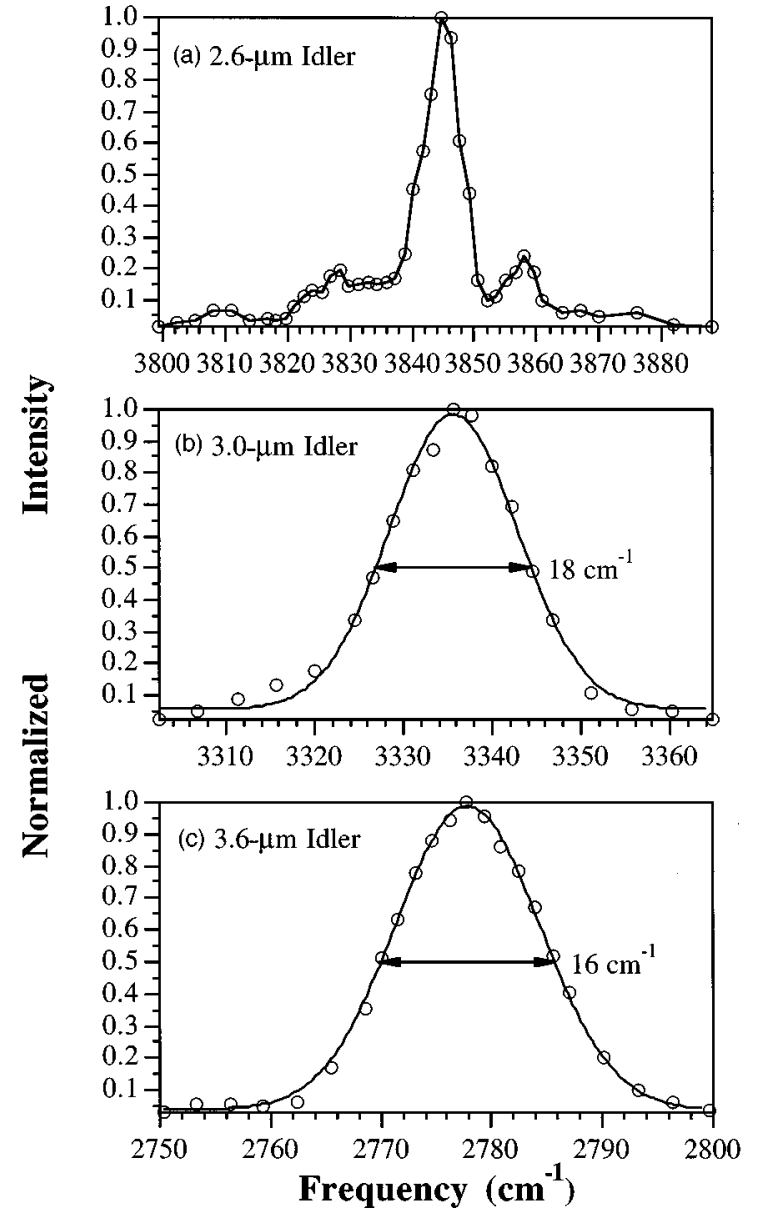

Fig. 5. Spectra of the idler beam at various wavelengths: (a) $2.6 \mu \mathrm{m}$, (b) $3.0 \mu \mathrm{m}$, and (c) $3.6 \mu \mathrm{m}$. Solid curves are a guide for the eye.

0.62 at 3.0 and $3.6 \mu \mathrm{m}$, respectively. As with the signal pulses, these are smaller than for $\operatorname{sinc}^{2}$ pulses but are approximately twice transform limited for Gaussian or sech ${ }^{2}$ pulses. Note also that there are no readily apparent sidelobes in the cross correlations.

Some rather interesting behavior occurs in the region near $2.6 \mu \mathrm{m}$. Water vapor is known to absorb in this region and causes significant disruption of the spatial and the temporal characteristics of the idler beam as it propagates through several feet of room air before being measured. The spectrum shown in the top panel of Fig. 5 shows a relatively narrow (ca. $10-\mathrm{cm}^{-1}$ FWHM) central peak riding on an irregular background extending over 80 $\mathrm{cm}^{-1}$. The corresponding cross correlation is shown in the top panel of Fig. 6 . Note the asymmetry and the leading peak (ca. 5-ps FWHM) followed by a relatively long decay (tens of picoseconds). Interestingly, these temporal and spectral profiles are actually a considerable improvement over the behavior observed before the pump bandwidth was narrowed from $21 \mathrm{~cm}^{-1}$ to $17 \mathrm{~cm}^{-1}$ (idler bandwidths decreased from $25-30 \mathrm{~cm}^{-1}$ to $16-18 \mathrm{~cm}^{-1}$ ). Two corresponding sets of measurements are shown in Fig. 7. The spectra exhibit similarly placed features, but the side peaks and background are larger relative to the main peak in the case of the spectrally broader pump.
Likewise, the cross correlations are similar except for a relative enhancement of the long decaying portion with respect to the central peak, which seems to occur on similar time scales. One possible explanation for this behavior is that a spectrally broad short pulse may coherently excite an ensemble of molecules. These molecules then undergo some sort of free-induction decay, giving rise to the decaying tails observed in the cross correlations and the structure observed in the spectra. As the idler-pulse bandwidth is decreased, the size of the excited ensemble diminishes, thereby decreasing the amplitude of the decaying portion of the cross correlation and the intensity of the spectral structure. This phenomena was not investigated in great detail.

The spatial characteristics of the idler beam were measured very carefully. The final idler beam was focused by $\mathrm{a}+125$-mm focal-length $\mathrm{CaF}_{2}$ lens, and a series of knifeedge measurements were performed. In this way a set of values for $\omega$ versus propagation distance was generated and fit to the equation ${ }^{21}$ that is shown along with the data in Fig. 8. The fit yields a value of $M^{2}$ of 3.45 , implying that the idler beam is approximately 3.5 times diffraction limited.

Figure 9(a) shows the experimental power curves for
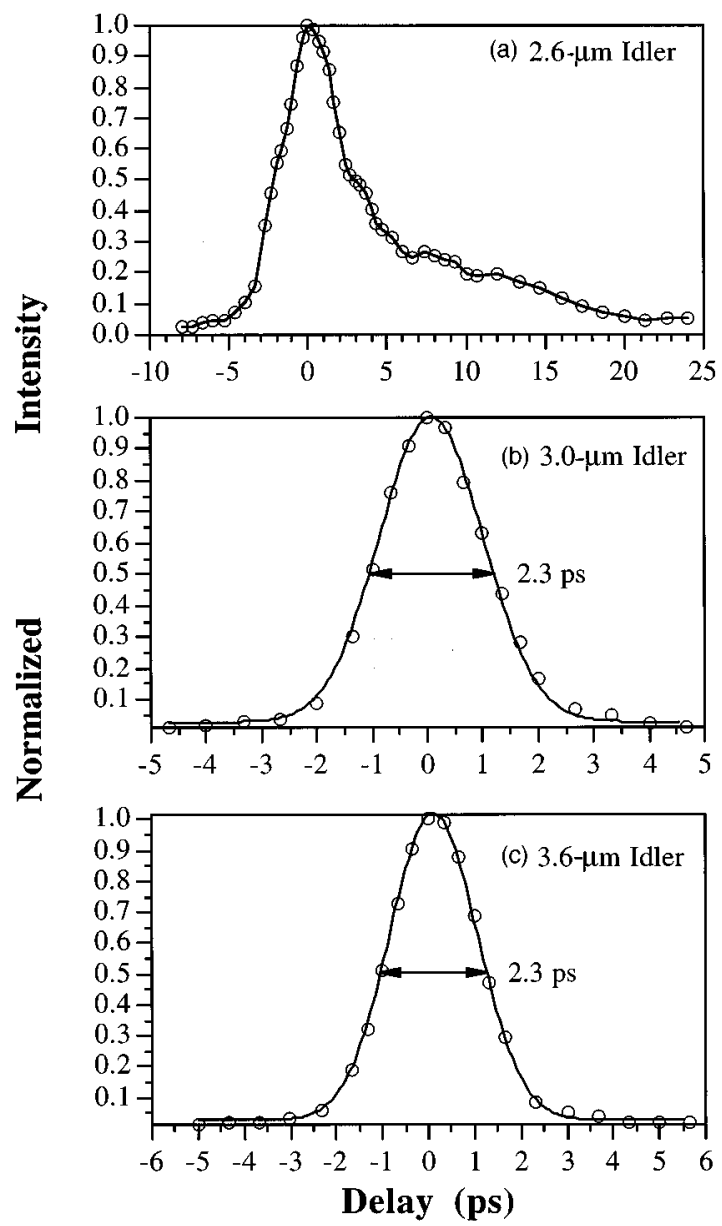

Fig. 6. Cross correlation of the idler beam at various wavelengths: (a) $2.6 \mu \mathrm{m}$, (b) $3.0 \mu \mathrm{m}$, and (c) $3.6 \mu \mathrm{m}$. Solid curves are a guide for the eye. 


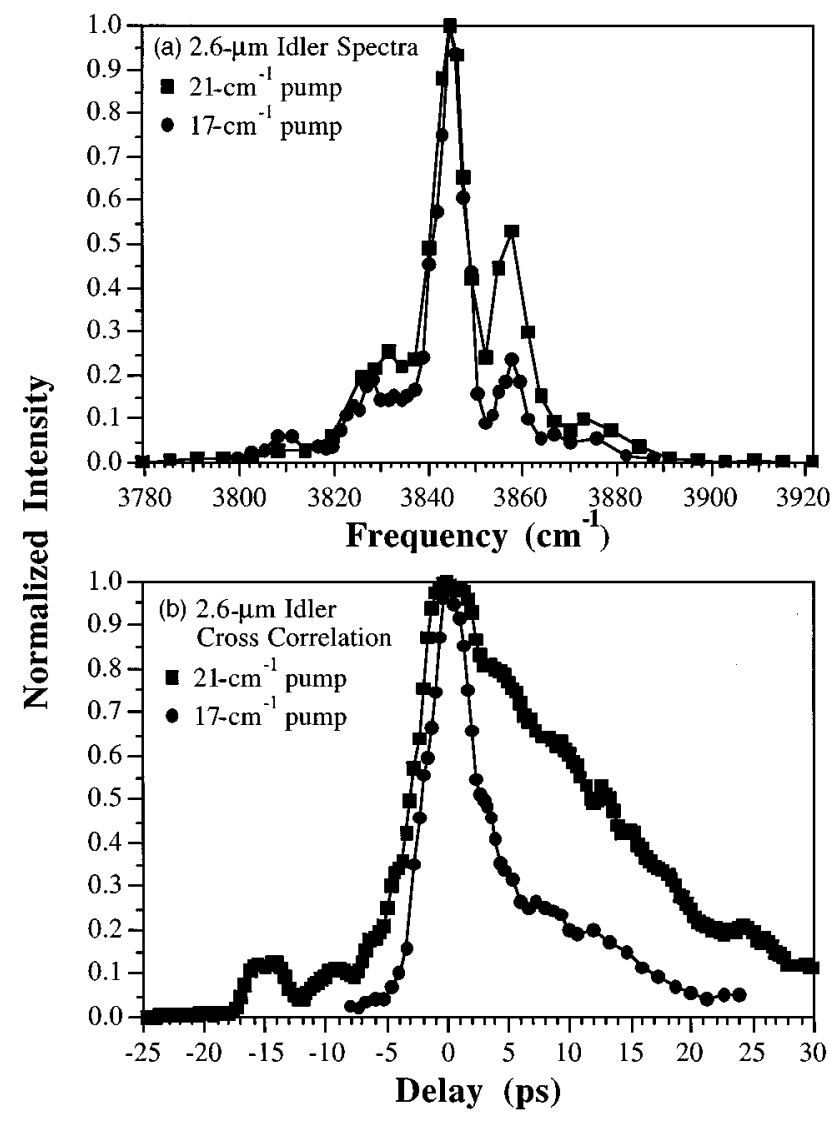

Fig. 7. Comparison of the spectra and the cross correlation of the idler beam in the water absorption region $(2.6 \mu \mathrm{m})$ for different pump spectral bandwidths: (a) spectra and (b) cross correlation.

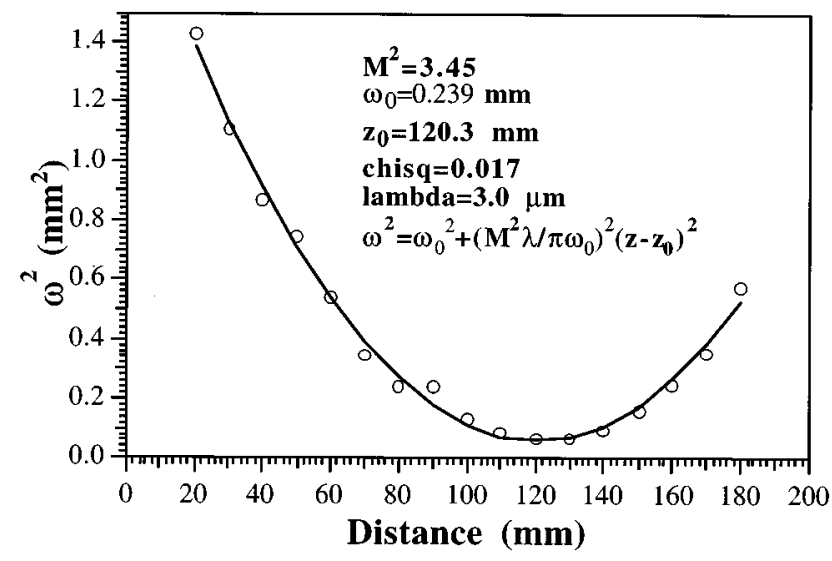

Fig. 8. Transverse spatial beam profile (see text) of the idler beam at $3.0 \mu \mathrm{m}$. Open circles are experimental data points, and the solid curve is a fit to $\omega^{2}=\omega_{0}^{2}+\left(M^{2} \lambda / \pi \omega_{0}\right)^{2}\left(z-z_{0}\right)^{2}$.

both the signal and the idler over the experimental tuning range. The energies reported were corrected for filter losses at the position of F3, and thus the idler energy available for SFG experiments is slightly lower. The idler and the signal energies are relatively flat at approximately 10 and $20 \mu \mathrm{J}$, respectively, with the exception of two features. The first feature is a dip in the idler energy at $2.7 \mu \mathrm{m}$, which is caused by absorption of $\mathrm{H}_{2} \mathrm{O}$ vapor in air, as discussed above (note that there is no corresponding dip in the signal energy). The second feature is a peak in both the idler and the signal energies at 3.6 and $1.04 \mu \mathrm{m}$, respectively, which is caused by three interacting effects. First, the intensity of the seed from the white light is increasing dramatically as the signal approaches $800 \mathrm{~nm}$. Second, the transmission of the dichroic mirror M4 is decreasing as the signal wavelength approaches 1.0 $\mu \mathrm{m}$. Finally, the value of $d_{\text {eff }}$ for type II phase matching in the $x z$ plane decreases as one goes to smaller angles according to $d_{\text {eff }}=d_{24} \sin \theta$, and thus the conversion efficiency decreases. The competing effect is the creation of a peak in both the idler and the signal energies. Figure 9(b) shows the photon conversion efficiency for both the signal and the idler corresponding to the energies in Fig. 9(a). The photon conversion efficiency was calculated with the energies from Fig. 9(a) and the total 800-nm pump energy (white light plus OPA1 plus OPA2 equal to $390 \mu \mathrm{J})$ for each wavelength. Thus the reported efficiencies are an overall efficiency of the entire system and not an individual efficiency for the OPA process in a KTP crystal. From this graph one can see that a total efficiency (signal plus idler) as high as $20 \%$ is achieved.

\section{SUM-FREQUENCY-GENERATION SPECTRA}

The glass/octadecyltrichlorosilane (OTS)/air interface has been extensively studied with $\mathrm{SFG},{ }^{28-30}$ and we use it here as a comparative test for our laser system. GuyotSionnest et al. have reported three peaks in the $\mathrm{C}-\mathrm{H}$ spectral region, all of which can be assigned to $\mathrm{C}-\mathrm{H}$ stretches of the terminal $\mathrm{CH}_{3}$ group. The first peak lies at $2878 \mathrm{~cm}^{-1}$ with a bandwidth of $7 \mathrm{~cm}^{-1}$ and has been assigned to the symmetric $(\mathrm{s})$ stretch. A second peak at $2942 \mathrm{~cm}^{-1}$ with a bandwidth of $9 \mathrm{~cm}^{-1}$ has been attributed to the Fermi resonance between the $s$ stretch and the overtone of the $\mathrm{C}-\mathrm{H}$ bending mode. A third peak at $2964 \mathrm{~cm}^{-1}$ has been assigned to the two nearly degenerate $\left(d_{x}\right.$ at $2969 \mathrm{~cm}^{-1}$ and $d_{y}$ at $\left.2958 \mathrm{~cm}^{-1}\right)$ asymmetric (d) stretches of the terminal $\mathrm{CH}_{3}$ group with bandwidths of 9 $\mathrm{cm}^{-1}$ each. Further, under $P_{\mathrm{sf}} P_{\text {vis }} P_{\text {ir }}$ polarization conditions the asymmetric (d) stretch and the symmetric (s) stretch were present with the Fermi resonance obscured by the asymmetric (d) stretch. Under $S_{\text {sf }} S_{\text {vis }} P_{\text {ir }}$ polarization conditions, only the symmetric (s) and Fermi resonance were present.

Figure 10 shows the SFG spectra of a saturated monolayer of OTS on glass in air under different polarization conditions obtained with our laser system. Each spectrum was generated by averaging 800 laser shots at each point in $0.002-\mu \mathrm{m}$ increments. With the $1-\mathrm{kHz}$ repetition rate of our system this was easily achieved in scans that lasted approximately $40 \mathrm{~min}$. Figure 10(a) shows the SFG spectrum under $P_{\mathrm{sf}} P_{\text {vis }} P_{\text {ir }}$ polarization conditions from our laser system. The asymmetric (d) stretch is the dominant peak present and occurs at $2964 \mathrm{~cm}^{-1}$ with a width of $21 \mathrm{~cm}^{-1}$. One can also see a small peak at $2879 \mathrm{~cm}^{-1}$ that is due to the symmetric (s) stretch and a shoulder at $2943 \mathrm{~cm}^{-1}$ that is due to the Fermi reso- 

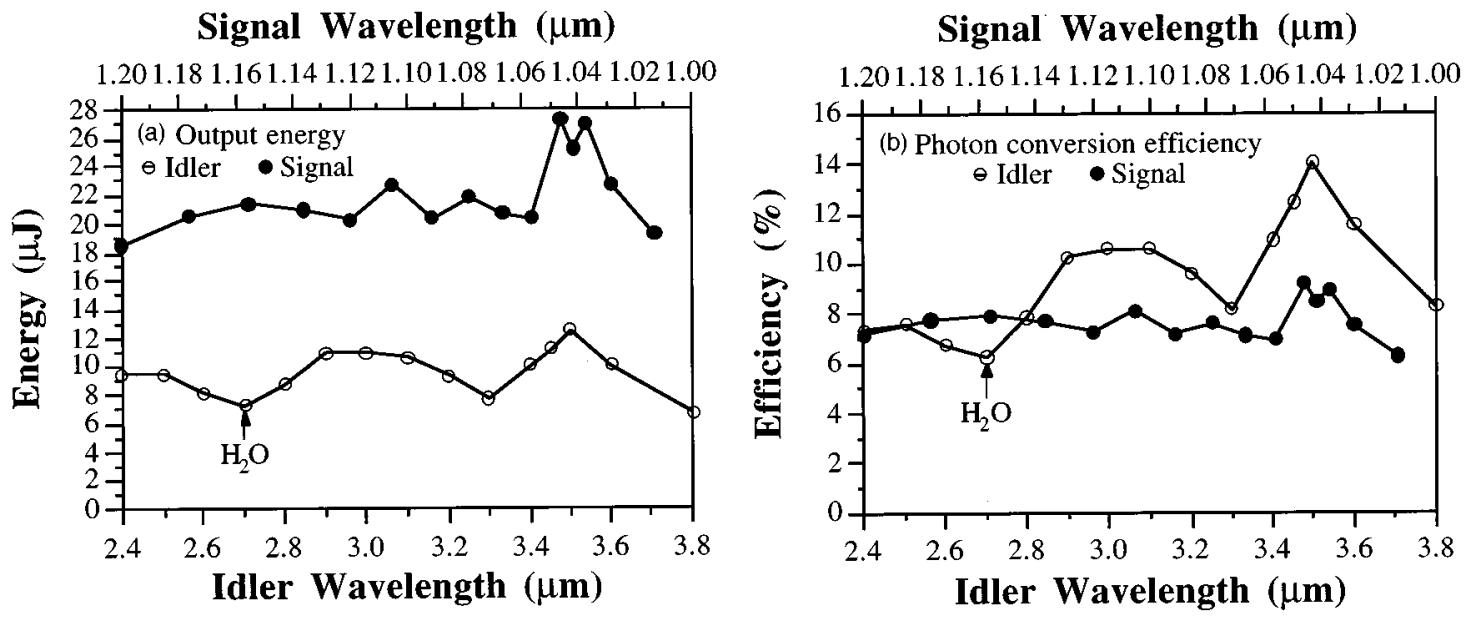

Fig. 9. Pulse energy and photon conversion efficiency for signal and idler beams: (a) output energy and (b) photon conversion efficiency. Solid curves are a guide to the eye.

nance. This is what is expected since the $P_{\text {sf }} P_{\text {vis }} P_{\text {ir }}$ polarization condition contains all the $\chi$ elements. Figure 10(b) shows the SFG spectrum under $S_{\mathrm{sf}} S_{\text {vis }} P_{\text {ir }}$ polarization conditions. For this case the symmetric (s) stretch

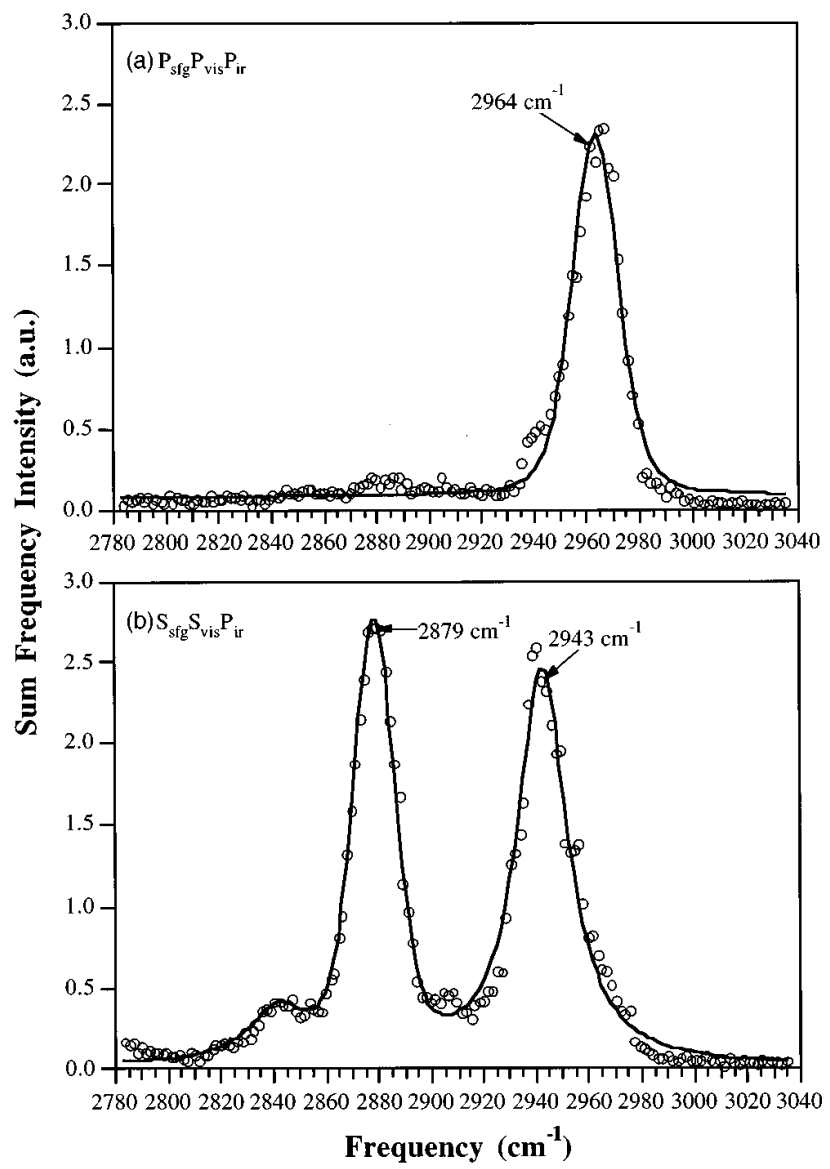

Fig. 10. Sum-frequency spectra of a monolayer of OTS on glass in air under different polarization conditions: (a) $P_{\text {sfg }} P_{\text {vis }} P_{\text {ir }}$ and (b) $S_{\text {sfg }} S_{\text {vis }} P_{\text {ir }}$. Solid curves are a guide to the eye. occurring at $2879 \mathrm{~cm}^{-1}$ with a width of $19 \mathrm{~cm}^{-1}$ and the Fermi resonance occurring at $2943 \mathrm{~cm}^{-1}$ with a width of $23 \mathrm{~cm}^{-1}$ are the dominant peaks present. The bandwidths of the individual peaks can be found by deconvolving the SFG widths with the $17-\mathrm{cm}^{-1}$ Gaussian laser bandwidth by assuming a Lorentzian vibration width. From this we get bandwidths of $4 \mathrm{~cm}^{-1}, 10 \mathrm{~cm}^{-1}$, and 7 $\mathrm{cm}^{-1}$ for the symmetric (s), Fermi resonance, and asymmetric (d) modes, respectively.

\section{CONCLUSION}

We have demonstrated that intense broadly tunable IR pulses are produced by this system. Further, the spatial, the spectral, and the temporal qualities of the IR beam make it an attractive source for nonlinear spectroscopic applications. The moderately narrow bandwidths and the broad tuning range will allow for the study of a large number of condensed phase interfacial molecules by means of their vibrational modes. The $16-18 \mathrm{~cm}^{-1}$ bandwidths reported here are well suited for studies of this type in which the bandwidths of the molecular vibrations are relatively large. Our tuning range spans and IR region in which molecules containing $\mathrm{OH}, \mathrm{NH}$, and $\mathrm{CH}$ bonds can be studied. We have demonstrated the ability to obtain SFG spectra in the $\mathrm{CH}$ stretching region with studies on the glass/OTS/air interface. We have also conducted preliminary SFG experiments on alcohol/air and $n$-alkane/air interfaces with great success in the $\mathrm{CH}$ stretch region. Water absorption of the IR beam as it propagates through air interfered with our ability to obtain spectra in the $\mathrm{OH}$ region from the glass/OTS/air and the alcohol/air interfaces. We are in the process of eliminating this limitation by removing the water in the beam path and further reducing the IR bandwidth. The practical limit of the bandwidths attainable by modifying the regenerative amplifier system is $5-10 \mathrm{~cm}^{-1}$, which would improve the resolution somewhat and virtually eliminate the water absorption effects. We are also in the process of extending the tuning range beyond $4.0 \mu \mathrm{m}$ so that we 
can study a wider variety of molecules. We will employ other nonlinear crystals such as $\mathrm{AgGaS}_{2}$ and $\mathrm{KNbO}_{3}$ to achieve tunability beyond $4.0 \mu \mathrm{m}$.

\section{ACKNOWLEDGMENT}

This research is supported by the Office of Naval Research.

\section{REFERENCES}

1. Q. Du, E. Freysz, and Y. R. Shen, "Surface vibrational spectroscopic studies of hydrogen bonding and hydrophobicity," Science 264, 826 (1994).

2. M. C. Mesmer, J. C. Conboy, and G. L. Richmond, "Observation of molecular ordering at the liquid-liquid interface by resonant sum frequency generation," J. Am. Chem. Soc. 117, 8039 (1995)

3. C. D. Bain, P. B. Davies, and R. N. Ward, "In situ sumfrequency spectroscopy of sodium dodecyl sulfate and dodecanol coadsorbed at a hydrophobic surface," Langmuir $\mathbf{1 0}$ 2060 (1994)

4. V. Pflumio, J. C. Vallet, A. J. Boeglin, A. A. Villaeys, and J. P. Lavoine, "Application of the sum-frequency generation to the vibrational spectroscopy of a Langmuir-Blodgett film," Phys. Rev. A 51, 3174 (1995).

5. K. Wolfrum, H. Graener, and A. Laubereau, "Sumfrequency vibrational spectroscopy at the liquid-air interface of methanol water solutions," Chem. Phys. Lett. 213 41 (1993)

6. D. Zhang, J. H. Gutow, K. B. Eisenthal, and T. F. Heinz, "Sudden structural change at an air/binary liquid interface: sum-frequency study of the air/acetonitrilewater interface," J. Chem. Phys. 98, 5099 (1993).

7. R. Laenen, K. Wolfrum, A. Seilmeier, and A. Laubereau, "Parametric generation of femtosecond and picosecond pulses for spectroscopic applications," J. Opt. Soc. Am. B 10, 2151 (1993).

8. H. M. van Driel and G. Mak, "Femtosecond pulses from the ultraviolet to the infrared: optical parametric processes in a new light," Can. J. Phys. 71, 47 (1993).

9. J. Y. Zhang, J. Y. Huang, Y. R. Shen, and C. Chen, "Optical parametric generation and amplification in barium borate and lithium triborate crystals," J. Opt. Soc. Am. B 10, 1758 (1993).

10. H. Vanherzeele, "Picosecond laser system continuously tunable in the 0.6-4 $\mu \mathrm{m}$ range," Appl. Opt. 29, 2246 (1990).

11. H. J. Krause and W. Daum, "High-power source of coherent picosecond light pulses tunable from 0.41 to $12.9 \mu \mathrm{m}$," Appl. Phys. B 56, 8 (1993).

12. U. Sukowski and A. Seilmeier, "Intense tunable picosecond pulses generated by parametric amplification in barium betaborate," Appl. Phys. B 50, 541 (1990).

13. F. Seifert, V. Petrov, and M. Woerner, "Solid-state laser system for the generation of midinfrared femtosecond pulses tunable from 3.3 to $10 \mu \mathrm{m}$," Opt. Lett. 19, 2009 (1994).

14. P. Hamm, C. Lauterwasser, and W. Zinth, "Generation of tunable subpicosecond light pulses in the midinfrared between 4.5 and $11.5 \mu \mathrm{m}$," Opt. Lett. 18, 1943 (1993).

15. S. Takeuchi and T. Kobayashi, "Broadband near-infrared pulse generation $\mathrm{KTiOPO}_{4}$," J. Appl. Phys. 75, 2757 (1994)

16. M. K. Reed, M. K. Steiner-Shepard, and D. K. Negus, "Widely tunable femtosecond optical parametric amplifier at $250 \mathrm{kHz}$ using a Ti:sapphire regenerative amplifier," Opt. Lett. 19, 1973 (1994).

17. M. K. Reed, M. K. Steiner-Shepard, M. S. Armas, and D. K Negus, "Microjoule-energy ultrafast optical parametric amplifiers," J. Opt. Soc. Am. B 12, 2229 (1995).

18. W. H. Knox, M. C. Downer, R. L. Fork, and C. V. Shank, "Amplified femtosecond optical pulses and continuum generation at 5-kHz repetition rate," Opt. Lett. 9, 552 (1984).

19. V. Petrov and F. Noack, "Tunable femtosecond optical parametric amplifier in the mid-infrared with narrow-band seeding," J. Opt. Soc. Am. B 12, 2214 (1995)

20. A. Yariv, Optical Electronics (Holt, Rinehart, \& Winston, New York, 1986).

21. A. E. Siegman, "Defining and measuring laser beam quality," in Solid State Lasers: New Developments and Applications, M. Inguscio and R. Wallenstein, eds. (Plenum, New York, 1993), p. 13

22. M. M. Wefers and K. A. Nelson, "Ultrafast optical waveforms," Science 262, 1381 (1993).

23. A. M. Weiner, D. E. Leaird, J. S. Patel, and J. R. Wullert, "Programmable femtosecond pulse shaping by use of a multielement liquid-crystal phase modulator," Opt. Lett. 15, 326 (1990).

24. G. R. Fleming, Chemical Applications of Ultrafast Spectroscopy (Oxford U. Press, New York, 1986).

25. D. E. Gragson, D. S. Alavi, and G. L. Richmond, "Tunable picosecond infrared laser system based on parametric amplification in KTP with a Ti:sapphire amplifier," Opt. Lett. 20, 1991 (1995).

26. V. G. Dmitriev, G. G. Gurzadyan, and D. N. Nikogosyan, Handbook of Nonlinear Optical Crystals (Springer-Verlag, New York, 1991).

27. H. Vanherzeele, J. D. Bierlein, and F. C. Zumsteg, "Index of refraction measurements and parametric generation in hydrothermally grown potassium titanyl phosphate (KTiOPO4)," Appl. Opt. 27, 3314 (1988).

28. P. Guyot-Sionnest, R. Superfine, J. H. Hunt, and Y. R Shen, "Vibrational spectroscopy of a silane monolayer at air/solid and liquid/solid interfaces using sum-frequency generation," Chem. Phys. Lett. 144, 1 (1988).

29. N. Watanabe, H. Yamamoto, A. Wada, K. Domen, C. Hirose, T. Ohtake, and N. Mino, "Vibrational sum-frequency generation (VSFG) spectra of $n$-alkyltrichlorosilanes chemisorbed on quartz plate," Spectrochim. Acta 50A, 1529 (1994).

30. K. Wolfrum, J. Lobau, and A. Laubereau, "Sum-frequency spectroscopy of physisorbed and chemisorbed molecules at liquid and solid surfaces using bandwidth limited picosecond pulses,” Appl. Phys. A 59, 605 (1994). 